\title{
Cross-cultural validation of an expanded Exercise Addiction Inventory: A preliminary protocol report
}

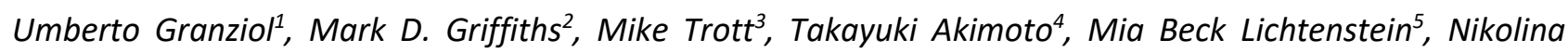
Bjegovic $^{1}$, Abril Cantú-Berrueto ${ }^{6}$, Brian Cook ${ }^{7}$, Ricardo de la Vega Marcos ${ }^{8}$, Zsolt Demetrovics ${ }^{9,10}$, Laura Di Lodovico $^{11}$, Nataliya Dmytrenko ${ }^{1}$, Alexei Egorov ${ }^{12,13}$, Olga V. Felsendorff ${ }^{14}$, Ruth Jiménez Castuera ${ }^{15}$, Elena $K_{\text {Khvatova }}{ }^{16}$, Emilio Landolfi ${ }^{17}$, Angelica Larios Delgado ${ }^{18}$, Marta Leyton Román ${ }^{19}$, Anthony Maher ${ }^{20}$, Liudmyla Mova $^{21}$, Robert Portman ${ }^{21}$, Antonio Rosado ${ }^{23}$, Dahiana Salazar Gonzále ${ }^{24}$, Melanie Schipfer ${ }^{25}$, Marco Solmi ${ }^{26}$, Oliver Stoll ${ }^{25}$, Paula Teixeira Fernandes ${ }^{27}$, Peiying Yang ${ }^{28}$, Liye Zou ${ }^{28}, *$ Attila Szabo ${ }^{9,29^{*} a}$

Funding: No financial support was received for this study. ZD's and AS' contribution was supported by the Hungarian National Research, Development and Innovation Office (KKP126835, K134807).

Conflict of interest: The authors have no conflict of interest to declare.

Ethics: The procedure described in this study protocol will be carried out in accordance with the Declaration of Helsinki. All participants provide informed consent for study participation. An umbrella protocol obtained formal authorization by one of the European Institutional Review Board (IRB). Co-authors outside the EU asked approval, when necessary, to their IRB. Participants will not be reimbursed for their participation.

Acknowledgments: The present work has been carried out in line with the aims of the research program "Dipartimenti di Eccellenza" from MIUR to the Department of General Psychology, University of Padua, Italy

* Corresponding author: Prof. Dr. Attila Szabo, Institute of Health Promotion and Sport Sciences, ELTE Eötvös Loránd University, 1225 Budapest, Prielle Kornélia Street 47, $3^{\text {rd }}$ Floor, Hungary, Email: szabo.attila@ppk.elte.hu

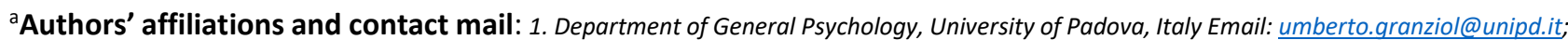
nataliya.dmytrenko@gmail.com; nikolina.bjegovic@studenti.unipd.it; 2. Psychology Department, Nottingham Trent University, UK. Email: mark.griffiths@ntu.ac.uk; 3.Vision and Eye Research Institute, Faculty of Health, Education, Medicine, and Social Care, Anglia Ruskin University, United Kingdom. Email: mike.trott@aru.ac.uk; 4. Faculty of Sport Sciences, Waseda University, Japan. Email:axi@waseda.jp; 5. Department of Clinical Research, University of Southern Denmark, Odense, Denmark. Email:mlichtenstein@health.sdu.dk; 6. University Autonomous of Nuevo León (UANL), France.Email:abrilcantubrr@uanl.edu.mx; 7.Private practice.Email: BrianCookPhd@gmail.com 8. Department of Physical Education, Sport \& Human Movement, Autonomous University of Madrid, Spain. Email:delavegaricardo@hotmail.com; 9. Institute of Psychology, ELTE Eötvös Loránd University, Budapest, Hungary. Email: demetrovics.zsolt@ppk.elte.hu; 10. Centre of Excellence in Responsible Gaming, University of Gibraltar, Gibraltar, Gibraltar. Email: zsolt.demetrovics@unigib.edu.gi; 11. Clinique des Maladies Mentales et de l'Encéphale, Hôpital Sainte-Anne, GHU Paris Psychiatrie et Neurosciences, Paris, France; Université de Paris, Institute of Psychiatry and Neuroscience of Paris (IPNP), INSERM U1266, Paris, France. laura.dilodovico@yahoo.com; 12. Department of Psychiatry and Addictions, St.Petersburg State University, Russia. Email:draegorov@mail.ru; 13. Laboratory of Behavior Neurophysiology and Pathology, I.M. Secenov Institute of Evolutionary Physiology and Biochemistry, St. Petersburg, Russia; 14. Faculty of Psychology, St. Petersburg University, Russia Email: olga.felsendorff@gmail.com; 15. Sport Sciences Faculty. University of Extremadura, Spain. Email: ruthii@unex.es; 16. Department of Psychology, Lesgaft Sport University, St.Petersburg, Russia. Email: elenakhvatskaya@mail.ru; 17. Faculty of Health Sciences, School of Kinesiology, University of the Fraser Valley, Canada. Email:Emilio.Landolfi@ufv.ca; 18. Asociación de Esgrima, National Autonomous University of Mexico (UNAM). Mexico. Email:angelicalarios13@gmail.com; 19. Rey Juan Carlos University, Spain. Email:marta.leyton@urjc.es; 20. Carnegie School of Education, Leeds Beckett University, United Kingdom. Email: anthony.maher@leedsbeckett.ac.uk; 21. Department of Modern Choreography, National University of Ukraine on Physical Education and Sport, Ukraine. Email: maluma@ua.fm; 22. Teesside University. Middlesborough, United Kingdom. Email:R.Portman@tees.ac.uk; 23. Faculty of Human Motricity, Lisbon University, Portugal. Email:arosado@fmh.ulisboa.pt; 24. Universidad Autónoma De Nuevo León, France. Email: dsalazargzz@hotmail.com; 25. Department of Sport Psychology and Sport Pedagogy, Institute of Sport Science, Martin-Luther-University Halle-Wittenberg, Germany. Email: melanie.schipfer@gmail.com; oliver.stoll@sport.uni-halle.de; 26. Department of Psychiatry, University of Ottawa, Ottawa, ON, Canada. Email: marco.solmi83@gmail.com; 27. Faculty of Physical Education, Department of Sports Science, Universidade Estadual de Campinas-UNICAMP, Brasil. Email: paula@fef.unicamp.br; 28. Body-Brain-Mind Laboratory, School of Psychology, Shenzhen University, Shenzhen 518060, China. Email: skyleryang40@gmail.com; liyezou123@gmail.com 29. Institute of Health Promotion and Sport Sciences, ELTE Eötvös Loránd University, Budapest, Hungary. Email: szabo.attila@ppk.elte.hu
} 


\section{ABSTRACT}

Background and aims. In the last 30 years, the continuously increasing number of studies investigating exercise addiction (EA) stimulated interest in developing instruments assessing the risk of exercise addiction (REA). One widely used tool is the Exercise Addiction Inventory (EAI) because it is a brief, easy-to-use, time-saving, and psychometrically validated tool. However, its items based on the 'components model of addiction' still lack some consistently reported reoccurring symptoms associated with exercise addiction. This protocol report outlines the methods of developing and validating an expanded version of the EAI (EAI-3) utilizing a large international sample.

Methods. The EAI-3 will be administered to over 5000 regular adult exercisers in 16 languages through an online survey. The survey will also include questions from the Exercise Dependence Scale-Revised, the Obsessive-Compulsive Inventory-Revised, the SCOFF questionnaire assessing susceptibility for eating disorders, and the Ten Item Personality Inventory (TIPI). The online study will investigate the factorial structure of the EAI-3 through exploratory and confirmatory factor analysis. Moreover, the study will test EAI-3's measurement invariance across languages and gender. Finally, the study aims to find a standard cutoff point for at-risk exercisers.

Expected results. The study is expected to obtain a good fit of the EAI-3 structure and general measurement invariance. In addition, associations are expected with another EA measure and the other measures of mental health assessed in the study helping to establish concurrent and divergent validity of the EAI-3. Preliminary results suggest good internal reliability for the EAI-3.

Discussion and conclusions. It is expected that the results will support an assessment tool useful in assessing the REA with accuracy and exhibiting reliability across gender and language (culture). Consequently, researchers will be able to assess the risk of exercise addiction more accurately.

Keywords: behavioral addictions; cultural differences; exercise dependence; measurement invariance; physical activity; questionnaire; validity

\section{INTRODUCTION}

\section{Background and rationale}

Since its first conceptualization (Baekeland, 1970), exercise addiction (EA) has become a growing interest among researchers, clinicians and physical health practitioners. Although not listed among behavioral addictions in diagnostic manuals, EA represents a physical and mental health concern among a minority of individuals. There are cases reported which show that EA can harm an individual's physical, mental, and social functioning (Hausenblas et al., 2017; Juwono \& Szabo, 2020), and it may even occur among regular sports practitioners (Lejoyeux et al., 2008), and young exercisers (Lichtenstein et al., 2018). Szabo (1995) suggested that EA is similar to other addictions. It can bias cognitive appraisals of events and decision-making processes, such as the attitude to 
continue practicing sport against medical advice (Granziol et al., 2021). Consequently, the need to better conceptualize its risk assessment and diagnosis is increasing.

Several psychometric scales have been developed to screen for the risk of exercise addiction (REA). The most widely used are the Exercise Dependence Scale-Revised (EDS-R; Hausenblas \& Symons Downs, 2002a, 2002b) and the Exercise Addiction Inventory (Terry et al., 2004). Both tools have good psychometric properties in several languages (Griffiths et al., 2015). The original EAl (Terry et al., 2004) has been validated in several languages, and it has been revised to obtain more valid scores (Szabo et al., 2019; Szabo, 2021). Still, some further refinements are needed; the EAI evaluates six components of addiction, namely salience, mood modification, tolerance, withdrawal symptoms, conflict, and relapse (Griffiths et al., 2005). However, EA is also strongly associated with feelings of guilt (Alcaraz-lbañez et al., 2021), the perseverance of potentially harmful exercise despite pain and injury (Griffiths, 1997; Granziol et al., 2021), and the negative consequences of addiction (Aidman \& Woollard, 2003; Juwono \& Szabo, 2020; Sicilia et al., 2020). The last is considered the cutoff criterion for establishing addiction (Juwono \& Szabo, 2020). Therefore, evaluating these behavioral aspects during the assessment could yield more accurate estimates of the REA. Moreover, assuming that an expanded model is practical in assessing the REA, it is essential to evaluate its structural invariance across gender and different languages/cultures.

\section{Primary study aims}

The present protocol describes the research plan aimed at evaluating:

a. The reliability and the factor structure of a new expanded version of the EAI named EAI-3.

b. The cross-culturally validity of and EAI-3 by testing its structural invariance across gender different languages/cultures.

c. The general cutoff point to screen individuals at high risk of EA and to examine whether this value is the same across gender and language.

\section{METHODS}

\section{Participants and sample size}

The present study includes experts from several international research groups working in: Brazil, Canada, China, Croatia, Denmark, France, Germany, Hungary, Italy, Japan, Mexico, Portugal, Russia, Serbia, Spain, Ukraine, the United Kingdom, and the United States of America (the involvement of researchers from Turkey is currently under way). The EAI-3 will be validated in 16 languages. Each country recruits approximately 400 participants, creating a total approximate sample consisting of $5,000-8,000$ individuals aged 18 years and over. To a priori estimate the sample size we estimated each subsample (for each statistical analysis) by using dedicated packages of the R environment ( $R$ core team, 2020) before adding up such sub-samples (see the "Statistical analysis section").

\section{Inclusion and exclusion criteria}




\begin{tabular}{|l|l|}
\hline Participants must be aged $\geq 18$ years. & Aged $<18$ years. \\
\hline $\begin{array}{l}\text { Participants must exercise at least three hours } \\
\text { per week. }\end{array}$ & $\begin{array}{l}\text { Participants exercise less than three hours per } \\
\text { week. }\end{array}$ \\
\hline $\begin{array}{l}\text { Participants must exercise for a minimum total } \\
\text { of } 150 \text { minutes every week. }\end{array}$ & $\begin{array}{l}\text { Participants exercise for less than a total of } 150 \\
\text { minutes every week. }\end{array}$ \\
\hline $\begin{array}{l}\text { Participants must exercise for at least six } \\
\text { months before participating in the study. }\end{array}$ & $\begin{array}{l}\text { Participants exercise for less than six months } \\
\text { before participating in the study. }\end{array}$ \\
\hline $\begin{array}{l}\text { Participants must be healthy (not reporting any } \\
\text { chronic illness). }\end{array}$ & Participants reported chronic illness. \\
\hline $\begin{array}{l}\text { Participants answered all the questions related } \\
\text { to exercise addiction. }\end{array}$ & $\begin{array}{l}\text { Participants do not answer all the questions } \\
\text { related to exercise addiction. }\end{array}$ \\
\hline
\end{tabular}

\section{Recruitment}

Participants are recruited online through calls on social media platforms (i.e., Facebook, Instagram, and Twitter). The recruitment targets online groups where sport or exercise performance is likely to be high. The snowball method (Kirchherr \& Charles, 2018) is also used to increase the potential pool of participants. The Qualtrics research platform ${ }^{2}$ (Qualtrics, 2021) is adopted for the study. Data collection is anonymous. The study is expected to end in the summer of 2022, but data collection will continue until the required sample size is obtained.

\section{Measures}

Participants will be asked to provide the following general information: gender; age; level of education; the level of competition/participation (i.e., regional national or international); years of practicing; hours of weekly practice; kind of sport practiced; the presence of neurological or mental health issues diagnosed by an expert, if and how the sports routine has changed due to the pandemic. Moreover, the following scales will be administered:

- Expanded Exercise Addiction Inventory (EAI-3): The EAI-3 items are evaluated on a 6-point Likert scale. In addition, to the original set of items on the EAI (Terry et al., 2004), three new items are added: "feelings of guilt," "training in case of injury," and "insight on negative consequences" (see Table 1 below), based on empirical evidence from the academic literature (Alcaraz-lbañez et al., 2021; Griffiths, 1997; Granziol et al., 2021; Juwono \& Szabo, 2020).

Table 1. Items of the EAI-3. The first six items are those of the original EAI (Terry et al., 2004). The last three items that expand the original EAI into EAI-3 are in italics. Each item will be rated on a 6-point agreement-disagreement scale.

\section{EAI-3 Text Items}

${ }^{2}$ https://psicologiapd.fra1.qualtrics.com/ife/form/SV 6Egp3Yd5npfODPO 


\begin{tabular}{|l|l|}
\hline Item 1 & Exercise is the most important thing in my life. \\
\hline Item 2 & $\begin{array}{l}\text { Concerns have arisen between me and my family and/or my partner about the } \\
\text { amount of exercise I do. }\end{array}$ \\
\hline Item 3 & I use exercise as a way of changing my mood (e.g., to get a buzz, to escape, etc.). \\
\hline Item 4 & Over time I have increased the amount of exercise I do in a day. \\
\hline Item 5 & If I have to miss an exercise session, I feel moody and irritable. \\
\hline Item 6 & $\begin{array}{l}\text { If I cut down the amount of exercise I do and then start again, I always end up } \\
\text { exercising as often as I did before. }\end{array}$ \\
\hline Item 7 & I feel guilty if I miss planned training or if my training does not go as well as planned. \\
\hline Item 8 & I am inclined to train when (or before completely recovered from) illness or injury. \\
\hline Item 9 & I have had physical, psychological and/or other issues due to my exercise regime. \\
\hline
\end{tabular}

- Exercise Dependence Scale-Revised (EDS-R; Hausenblas \& Downs, 2002a, 2002b). The EDS$\mathrm{R}$ comprises 21 questions rated on a 6-point Likert response scale. It has seven sub-scales: withdrawal, continuance, tolerance, control loss, a decrease of other activities, time, and effect of intention. This scale is used as a 'gold standard' to test the criterion validity of the EAI-3, since many studies showed its stability in investigating REA (Di Lodovico et al., 2017; Granziol et al., 2021).

- SCOFF (Morgan et al., 1999): The SCOFF is a dichotomous five-item scale used to screen people for the risk of developing an eating disorder. It has the advantage of being very short and with an explicit cutoff (total score $\geq 2$, interpreted as at-risk). This measure was included because eating disorders are often comorbid or primary to exercise addiction (AlcarazIbáñez et al., 2020; Trott et al., 2020).

- Obsessive-Compulsive Inventory-Revised (OCI-R; Abramowitz \& Deacon, 2006): The OCI-R comprises 18 items rated on a 5-point Likert scale. It assesses the tendency toward obsessions and compulsive behaviors, and comprises six factors: washing, obsessing, hoarding, ordering, checking, and mental neutralizing. This measure was included because of its relationship with EA (Gulker et al., 2020).

- The Ten-Item Personality Inventory (TIPI; Gosling et al., 2003): The TIPI comprises 10 items rated on a 7-point Likert scale. It assesses the big five personality domains: extroversion, agreeableness, openness to experience, conscientiousness, and emotional stability. This tool is used because personality factors have been associated with EA (Bircher et al., 2017).

\section{Procedure}

After obtaining consent, participants generate a personal alphanumeric code associated with their responses and use it to eliminate duplicate responses. Then, they answer blocks of questions in random order both within and between blocks. However, the first block of the online study, 
containing sociodemographic questions, is not randomized. The completion of the survey takes, on the average, 10 minutes. Too short completion times will be deleted from the data-analyses.

\section{Statistical analysis}

All the statistical analyses are based on the $R$ environment. For each sample size estimation, an $\alpha=$ .05 and a power of .80 is assumed. The SPSS software will also be used in various analyses.

\section{Cultural adaptation}

The new questions of the EAI-3 will be translated and back-translated by each collaborating group in 15 nations. The same procedure applies to all other dependent measures that are not validated in the participating nations.

\section{Factor structure}

After the recruitment ends, a confirmatory factor analysis (CFA) will then be performed on the nine items of the EAI-3. Both single and bifactor models will be tested. Depending on the item skewness, the appropriate estimator will be used. In accord with the general practice, the following goodnessof-fit statistics have been selected: Comparative Fit Index (CFI), Root Mean Square Error of Approximation's (RMSEA), and Standardized Root Mean Square Residual (SRMR). By considering a model with 27 degrees of freedom, to obtain an RMSEA $\leq .04,547$ responses are needed $(\sim 37$ respondents per language). To understand if the new item changes the factorial structure of the EAI-3, an additional sample of 547 responses will be used also to perform, preliminary to CFA, an exploratory factor analysis. In case more than one factor will emerge, the models will be compared in terms of fit to data.

\section{Cross-cultural validation}

To cross-culturally validate the model, measurement invariance will be tested on the EAI-3 items' score, which will show whether participants' responses change as a function of their gender and language (i.e., $2 \times 15$ = 30 groups). Configural, metric, and scalar invariances will be focused upon. Whenever measurement non-invariance is found, partial invariance will also be tested. Approximately 1500 responses will be necessary ( $\sim 0$ respondents per language) to examine invariance. Samples size for factor structure and measurement invariance will be estimated with the semPower package (Moshagen \& Erdfelder, 2016).

\section{Reliability and cutoff}

The reliability will be tested using the Cronbach Alpha (applying ordinal items' adjustments, if necessary) and McDonald's Omega (McDonal, 1999). Composite reliability ( Netemeyer et al., 2003) will also be tested. Both the specificity and sensitivity of EAI-R will be tested through the receiver operating characteristic (ROC) curve. Finally, the study will use the score obtained at EDS-R as an external criterion for determining the optimal EAI-3 cutoff. Considering that the prevalence of the REA in previous studies using EAI with regular exercisers is approximately $3.4 \%$ (Marques et al., 2019), and setting an area under the curve equal to .80, the minimum sample size for each language/culture would be 147 respondents. This size was estimated using the easyROC shiny app 
(Goksuluk et al., 2016). Therefore, a total subsample of 2205 respondents is needed to estimate the optimal cutoff. It will be used also to estimate the reliability coefficients.

\section{Concurrent and convergent validity}

The study will test the correlations between the EAI-3 and EDS-R OCI-R, SCOFF, and TIPI scores using the Spearman correlation coefficient (unless the obtained data will be normally distributed). To obtain a correlation coefficient of at least . 50 , the study estimated 435 respondents ( 29 respondents per language or culture) through the pwr package (Champely, 2020). Therefore, the required subsample is 1740 respondents (i.e., 435 X 4). Apart from EAl-3 validation, several other hypotheses will be tested using regression analyses, chi-square, and multivariate statistics (see Table 2 below).

Table 2. Hypotheses planned to be tested from the data obtained in the present study.

\begin{tabular}{|c|c|c|}
\hline Research Question & $\begin{array}{l}\text { Type of } \\
\text { study }\end{array}$ & Hypothesis \\
\hline $\begin{array}{l}\text { Are the team and competitive exercises more } \\
\text { closely connected to EA-linked eating disorder } \\
\text { symptoms than individual and non-competitive } \\
\text { exercisers? }\end{array}$ & CS & $\begin{array}{l}\text { Individual exercisers will exhibit more } \\
\text { eating disorders in the context of the } \\
\text { REA }\end{array}$ \\
\hline $\begin{array}{l}\text { Is there a cross-cultural difference in EA, its } \\
\text { prevalence, and related dysfunctions based on } \\
\text { SCOFF and OCI-R? }\end{array}$ & CS & $\begin{array}{l}\text { Cultural differences will exist in the type } \\
\text { and strength of the relationships. }\end{array}$ \\
\hline $\begin{array}{l}\text { Is there a difference in EA and associated } \\
\text { personality measures in different sports and } \\
\text { exercises (for example, team/individual, } \\
\text { competitive /non-competitive)? }\end{array}$ & CS & $\begin{array}{l}\text { Differences related to personality will } \\
\text { be expected based on past research } \\
\text { (i.e., team sports athletes are more } \\
\text { extroverted) }\end{array}$ \\
\hline $\begin{array}{l}\text { Are eating disorders, obsessive-compulsive } \\
\text { symptomatology, and personality traits similarly } \\
\text { associated with EA across cultures? }\end{array}$ & CS & $\begin{array}{l}\text { Cross-cultural differences will exist in } \\
\text { the relationship between EA and the } \\
\text { other dependent measures. }\end{array}$ \\
\hline $\begin{array}{l}\text { Which kind of personality structure is more prone } \\
\text { to EA? Is the finding consistent across the nations? }\end{array}$ & CS & $\begin{array}{l}\text { High traits of openness to experience } \\
\text { and low emotional stability will be } \\
\text { related to REA. }\end{array}$ \\
\hline $\begin{array}{l}\text { Did the pandemic reduce the amount of sport in a } \\
\text { different way between at-risk and non-at-risk of } \\
\text { EA? }\end{array}$ & CS & $\begin{array}{l}\text { A significant reduction will be expected } \\
\text { in the high REA groups only. }\end{array}$ \\
\hline $\begin{array}{l}\text { Is the EA independent of the reason for exercise } \\
\text { (i.e., health skill, social), and do the findings agree } \\
\text { in } 15 \text { nations? }\end{array}$ & CS & $\begin{array}{l}\text { Health-oriented exercisers will exhibit } \\
\text { higher EA across the } 15 \text { nations. }\end{array}$ \\
\hline Which is the most central component of EA? & $\begin{array}{l}\text { Network } \\
\text { study }\end{array}$ & $\begin{array}{l}\text { Salience could be the most central } \\
\text { component. }\end{array}$ \\
\hline $\begin{array}{l}\text { The relationship among EA, Eating disorders, and } \\
\text { OCD. Can it be modeled as a network? }\end{array}$ & $\begin{array}{l}\text { Network } \\
\text { study }\end{array}$ & Yes, with a central role of EA. \\
\hline
\end{tabular}




\begin{tabular}{|l|l|l|}
$\begin{array}{l}\text { Is the EAI-3 valid and invariant in team/individual } \\
\text { sports and competing/non-competing exercisers? }\end{array}$ & CS & $\begin{array}{l}\text { The structure of EAl-3 will be invariant } \\
\text { across the type of sport (team vs. } \\
\text { individual) or exercisers competing and } \\
\text { competing. }\end{array}$ \\
\hline $\begin{array}{l}\text { Is EAI-3 cutoff the same across the form of exercise } \\
\text { (team/individual) and level of exercise } \\
\text { involvement (competitive/non-competitive)? }\end{array}$ & CS & The cutoff will be the same. \\
\hline
\end{tabular}

CS: Cross-sectional; EA: Exercise addiction; EAI: Exercise Addiction Inventory

\section{Ethics}

The procedure described in this study protocol will be carried out in accord with the Declaration of Helsinki. Participants have to provide their informed consent for participation. An umbrella protocol obtained formal authorization by one of the European Institutional Review Board (IRB). Co-authors outside the EU ask for approval from their IRB. Participants will not be paid for their participation.

\section{RESULTS}

\section{Expected outcomes}

A single-factor structure of the EAI-3 is expected. In terms of cross-cultural validity, it is expected that the structure will obtain a good to excellent fit for each translated version and overall measurement invariance. Good reliability (e.g., $\alpha \geq .75$ ) is expected as well as a cutoff reflecting an adequate sensitivity-specificity balance. Finally, meaningful and positive correlations are expected between EAI-3 and the other measures. Table 3 shows some preliminary and descriptive analyses.

Table 3A. Preliminary descriptive results based on fully completed data ( $n=736$ on December 25, 2021)

\begin{tabular}{|l|l|}
\hline Measures & Data \\
\hline Number of responses on December 25, 2021 & 736 \\
\hline Gender distribution & Male: 386; Female: 349; Other: 1 \\
\hline Mean (M) age \pm standard deviation (SD), range & $\mathrm{M}=36.6$ (SD = 14.1), range $18-75$ years \\
\hline Weekly frequency of exercise & $\mathrm{M}=4.4, \mathrm{SD}=1.8$, range $3-15$ occasions \\
\hline Duration of an average exercise session & $\mathrm{M}=81.1, \mathrm{SD}=35.5$, range $10-400$ minutes \\
\hline Exercise history (years) & $\mathrm{M}=18.0, \mathrm{SD}=12.8$, range $.83-60$ years \\
\hline Reason for exercise & Health = 503; Skill = 155; Social = 50; Miss. = 28 \\
\hline Form of exercise: competitive/non-competitive & Competitive = 420 ; Non-competitive = 316 \\
\hline Form of exercise: aerobic, anaerobic, both & aerobic = 168; anaerobic = 38; both = 510 \\
\hline Form of exercise: individual, team, both & individual = 435; team = 140; both = 161 \\
\hline Form of exercise: organized, self-scheduled & organized = 304; self-scheduled = 432 \\
\hline Most responses to date (country) & $\begin{array}{l}\text { Italy = 380; Spain = 109; Germany = 77; United } \\
\text { Kingdom = 48; Hungary = 38 }\end{array}$ \\
\hline
\end{tabular}




\begin{tabular}{|l|l|}
\hline Reliability of the 9-item EAI-3 (Cronbach's $\alpha$ ) & .81 \\
\hline Reliability of the original 6-item EAI & .74 \\
\hline
\end{tabular}

Table 3B. Some further preliminary results based on fully completed data on February 06, 2022

\begin{tabular}{|c|c|}
\hline Measures & Data \\
\hline Number of responses on December 25, 2021 & 1203 \\
\hline Gender distribution & Male: 593; Female: 607; Other: 3 \\
\hline Weekly exercise frequency & $M=4.49(S D=2.13)$ \\
\hline Exercise history (years) & $M=15.37(S D=12.48)$ \\
\hline Main reason for exercise & Health $=787$, Skill $=290$, Social $=78$ \\
\hline Form of exercise & Aerobic $=266$, Anaerobic $=92$, Mixed $=835$ \\
\hline Competition status & None $=332$, Amateur $=583$, Athlete $=277$ \\
\hline Competition level & Regional $=365$, National $=227$, Int' $^{\prime}=101$ \\
\hline Known illness & Yes $=188$, No $=1005$ \\
\hline Organized exercise/sport & Yes $=468$, Self-organized $=722$ \\
\hline Mean $(\mathrm{M})$ age \pm standard deviation $(\mathrm{SD})$, range & $M=33.75(S D=14.31)$, range $18-99$ years \\
\hline Prevalence of EA risk based on EAI original & $7.97 \%$ \\
\hline $\begin{array}{l}\text { Prevalence of EA risk based on EAI-3 top } 20 \% \\
\text { scores (tentative scoring based on original EAI) }\end{array}$ & $\begin{array}{l}5.6 \% \text { (this value will be different once a cut-off } \\
\text { point is calculated for the new EAI-3 }\end{array}$ \\
\hline Sex differences (male, female) in EAI and EAI-3 & None $(F(1,1138)=.18$ and $.02 ; N S)$ \\
\hline EA differences based on competition status & None $(F(2,661)=1.57$ and $1.69 ; \mathrm{NS})$ \\
\hline Correlation between exercise history and EA & EAI $r=.052, N S ; E A I-3=.101, p<.001$ \\
\hline Correlation between weekly exercise and EA & EAI $r=.152, p<.001 ;$ EAI-3 = .177, $p<.001$ \\
\hline Reliability of the original 6-item EAI & .73 \\
\hline Reliability of the 9-item EAI-3 (Cronbach's $\alpha$ ) & .80 \\
\hline
\end{tabular}

\section{Preliminary Principal Components Analysis (PCA)}

A preliminary scan-PCA supported the unidimensional (one component) nature of the nine EAI-3 items $\left(\mathrm{KMO}=.856, \chi_{(36)}=1615.01, p<.001\right)$, accounting for $40 \%$ of the variance. The scree plot also confirmed the one-factor solution.

\section{Data reporting and dissemination}

This study protocol will be followed until the necessary sample size is obtained. Several publications will emerge based on examining the specific hypotheses presented in Table 2, which is not exhaustive because numerous mediatory roles, such as age and gender, and the volume of exercise could be examined if the primary analyses warrant it. Publications will be aimed at sports science and psychology journals. 


\section{DISCUSSION}

The increase of EA observed in different countries and studies (Juwono and Szabo, 2020, Marques et al., 2019) suggests that EA may be a clinical condition with an occurrence higher than expected that must be assessed in a cross-cultural perspective. Furthermore, more reliable research is needed for establishing its clinical diagnosis criteria. The present study protocol tries to reach this goal by describing the details to perform a cross-cultural validation of the EAI-3. In addition, the current protocol is presented as a preprint to share with the scientific community the ongoing work of an international team consisting of nearly 30 scholars. Finally, there is a desire to illustrate how the study could simultaneously address several independent hypotheses, the results of which will be shared in specialized empirical journals (see Table 2 for a complete list).

\section{Challenges and limitations}

The study accounts for some challenges that could become severe limitations. For example, Juwono and Szabo (2020) suggested that EA appears to be more frequent among female exercisers due to their higher propensity and willingness to speak about the problem. Another issue could be the participant recruitment. Nowadays, there are too many competing online studies. Furthermore, the self-report responses will make it difficult to distinguish whether negative consequences resulting from high levels of EA are representative of maladaptive unintentional behavior or (in elite athletes) deliberate athletic training. Finally, cause-and-effect relationships cannot be determined in any of the hypothesis tests performed in the present study.

\section{Impact}

The study will try to generate a standard assessment model and a more sensitive tool through a new brief instrument that could be used independently of language, culture, and gender in exercisers aged 18 years or older. Hopefully, the results will yield a psychometric instrument that could, in future studies, differentiate the REA in the context of various exercise characteristics, such as team/individual sports, organized/self-scheduled sports, and competitive/non-competitive sports. Moreover, the EAI-3 will also consider symptoms related to EA but ignored in many studies to date. These symptoms include the sense of guilt, training when injured, and the perception of negative consequences of exercisers' training regimen. 


\section{References}

Abramowitz, J. S., \& Deacon, B. J. (2006). Psychometric properties and construct validity of the Obsessive-Compulsive Inventory-Revised: Replication and extension with a clinical sample. Journal of Anxiety Disorders, 20(8), 1016-1035.

Aidman, E. V., \& Woollard, S. (2003). The influence of self-reported exercise addiction on acute emotional and physiological responses to brief exercise deprivation. Psychology of Sport and Exercise, 4(3), 225-236. https://doi.org/10.1016/S1469-0292(02)00003-1

Alcaraz-Ibañez, M., Chiminazzo, J. G. C., Sicilia, A., \& Fernandes, P. T. (2021). Body and appearancerelated self-conscious emotions and exercise addiction in Brazilian adolescents: A personcentred study. Journal of Sports Sciences, 39(13), 1528-1536. doi: 10.1080/02640414.2021.1883290

Alcaraz-Ibáñez, M., Paterna, A., Sicilia, A. \& Griffiths, M. D. (2020). Morbid exercise behaviour and eating disorders: A meta-analysis. Journal of Behavioral Addictions, 9, 206-224.

Baekeland, F. (1970). Exercise deprivation: Sleep and psychological reactions. Archives of General Psychiatry, 22(4), 365-369. https://doi.org/10.1001/archpsyc.1970.01740280077014

Bircher, J., Griffiths, M. D., Kasos, K., Demetrovics, Z., \& Szabo, A. (2017). Exercise addiction and personality: a two-decade systematic review of the empirical literature (1995-2016). Baltic Journal of Sport and Health Sciences, 3(106), 19-33. doi:10.33607/bjshs.v3i106.30

Champely, S. (2020). pwr: Basic functions for power analysis. R package version 1.3-0. https://CRAN.R-project.org/package=pwr

Di Lodovico, L., Poulnais, S., \& Gorwood, P. (2019). Which sports are more at risk of physical exercise addiction: A systematic review. Addictive behaviors, 93, 257-262.

Goksuluk, D., Korkmaz, S., Zararsiz, G., \& Karaagaoglu, A. E. (2016). easyROC: an interactive webtool for ROC curve analysis using R language environment. $R$ Journal, 8(2), 213.

Gosling, S. D., Rentfrow, P. J., \& Swann Jr, W. B. (2003). A very brief measure of the Big-Five personality domains. Journal of Research in Personality, 37(6), 504-528.

Granziol, U., Zorzi, A., Cardaioli, F., Cipriani, A., D'Ascenzi, F., Firth, J., Stubbs, B., Trott, M., \& Solmi, M. (2021). Exercise addiction in athletes: Comparing two assessment instruments and willingness to stop exercise after medical advice. Psychological Assessment, 33(4), 326-337. https://doi.org/10.1037/pas000098" https://doi.org/10.1037/pas000098.

Griffiths, M.D. (1997). Exercise addiction: A case study. Addiction Research, 5, 161-168.

Griffiths, M.D. (2005). A 'components' model of addiction within a biopsychosocial framework. Journal of Substance Use, 10, 191-197. 
Griffiths, M. D., Szabo, A., \& Terry, R. (2005). The Exercise Addiction Inventory: A quick and easy screening tool for health practitioners. British Journal of Sports Medicine, 39(6), e30. doi:10.1136/bjsm.2004.017020.

Griffiths, M. D., Urbán, R., Demetrovics, Z., Lichtenstein, M. B., de la Vega, R., Kun, B., ... \& Szabo, A. (2015). A cross-cultural re-evaluation of the Exercise Addiction Inventory (EAI) in five countries. Sports Medicine-Open, 1(1), 1-7.

Gulker, M. G., Laskis, T. A., \& Kuba, S. A. (2001). Do excessive exercisers have a higher rate of obsessive-compulsive symptomatology? Psychology, Health \& Medicine, 6(4), 387-398.

Hausenblas, H. A., Schreiber, K., \& Smoliga, J. M. (2017). Addiction to exercise. BMJ (Clinical Research Ed.), 357, Article j1745. https://doi.org/10.1136/bmj.j1745

Hausenblas, H. A., \& Downs, S. D. (2002a). Exercise Dependence Scale-21 manual. http://www.personal.psu.edu/dsd11/eds/eds21manual.pdf.

Hausenblas, H. A., \& Downs, S. D. (2002b). How much is too much? The development and validation of the Exercise Dependence Scale. Psychology \& Health, 17(4), 387-404. https://doi.org/10.1080/0887044022000004894.

Kirchherr, J., \& Charles, K. (2018). Enhancing the sample diversity of snowball samples: Recommendations from a research project on anti-dam movements in Southeast Asia. PloS One, 13(8), e0201710. https://doi.org/10.1371/journal.pone.0201710

Juwono, I.D., \& Szabo, A. (2020) 100 cases of exercise addiction: More evidence for a widely researched but rarely identified dysfunction. International Journal of Mental Health and Addiction 19, 1799-1811. https://doi.org/10.1007/s11469-020-00264-6

Lejoyeux, M., Avril, M., Richoux, C., Embouazza, H., \& Nivoli, F. (2008). Prevalence of exercise dependence and other behavioral addictions among clients of a Parisian fitness room. Comprehensive Psychiatry, 49(4), 353-358. https://doi.org/10.1016/i.comppsych.2007.12.005

Lichtenstein, M. B., Griffiths, M. D., Hemmingsen, S. D., \& Støving, R. K. (2018). Exercise addiction in adolescents and emerging adults. Validation of a youth version of the Exercise Addiction Inventory. Journal of Behavioral Addictions, 7(1), 117-125.

https://doi.org/10.1556/2006.7.2018.01

Marques, A., Peralta, M., Sarmento, H., Loureiro, V., Gouveia, É. R., \& de Matos, M. G. (2019). Prevalence of risk for exercise dependence: A systematic review. Sports Medicine, 49(2), 319-330. https://doi.org/10.1007/s40279-018-1011-4

McDonald, R. P. (1999). Test theory: A unified treatment. Mahwah, NJ : Lawrence Erlbaum

Morgan, J. F., Reid, F., \& Lacey, J. H. (1999). The SCOFF questionnaire: assessment of a new screening tool for eating disorders. BMJ, 319(7223), 1467-1468. 
Moshagen, M., \& Erdfelder, E. (2016). A new strategy for testing structural equation models. Structural Equation Modeling, 23, 54-60. doi:10.1080/10705511.2014.950896.

Netemeyer, R. G., Bearden, W. O., \& Sharma, S. (2003). Scaling procedures: Issues and applications. Sage Publications.

Qualtrics Compani (2021). Qualtrics, Provo, UT, USA. https://www.qualtrics.com

Sicilia, A., Paterna, A., Alcaraz-lbáñez, M., \& Griffiths, M. D. (2021). Theoretical conceptualizations of problematic exercise in psychometric assessment instruments: A systematic review. Journal of Behavioral Addictions, 10, 4-20.

Szabo, A. (1995). The impact of exercise deprivation on well-being of habitual exercises. Australian Journal of Science and Medicine in Sport, 27(3), 68-75.

Szabo, A. (2021). Model fit and reliability of the Hungarian version of the Revised Exercise Addiction Inventory (EAI-R-HU). (2021). Mentálhigiéné És Pszichoszomatika, 22(4), 376-394. doi:10.1556/0406.22.2021.013, Retrieved from: https://akjournals.com/view/journals/0406/22/4/article-p376.xml

Szabo, A., Pinto, A., Griffiths, M. D., Kovácsik, R., \& Demetrovics, Z. (2019). The psychometric evaluation of the Revised Exercise Addiction Inventory: Improved psychometric properties by changing item response rating, Journal of Behavioral Addictions, 8(1), 157-161. Retrieved from: https://akjournals.com/view/journals/2006/8/1/article-p157.xml

Terry, A., Szabo, A., \& Griffiths, M. (2004). The exercise addiction inventory: A new brief screening tool. Addiction Research and Theory, 12(5), 489-499. https://doi.org/10.1080/16066350310001637363

Trott, M., Jackson, S. E., Firth, J., Fisher, A., Johnstone, J., Mistry, A., ... \& Smith, L. (2020). Exercise addiction prevalence and correlates in the absence of eating disorder symptomology: $A$ systematic review and meta-analysis. Journal of Addiction Medicine, 14(6), e321-e329. 